\title{
Catalytic and Magnetic Behaviors of Excessively Charged Silver, Copper, Platinum, and Rhodium Atomic Clusters
}

\author{
Shideh Ahmadi, ${ }^{* \dagger}$ Xi Zhang, ${ }^{\ddagger}$ Yinyan Gong, ${ }^{\S}$ Weiguang Zhu, ${ }^{\dagger}$ Chang Q. Sun ${ }^{\dagger}$ \\ 'NOVITAS, School of Electrical and Electronic Engineering, Nanyang Technological \\ University, 639798 Singapore, Singapore \\ ${ }^{\ddagger}$ Institute of Nanosurface Science and Engineering, Shenzhen University, Shenzhen 518060, \\ China \\ ${ }^{\S}$ Center for Coordination Bond and Electronic Engineering, College of Materials Science and \\ Engineering, China Jiliang University, Hangzhou 310018, China \\ *E-mail: shideh001@e.ntu.edu.sg
}



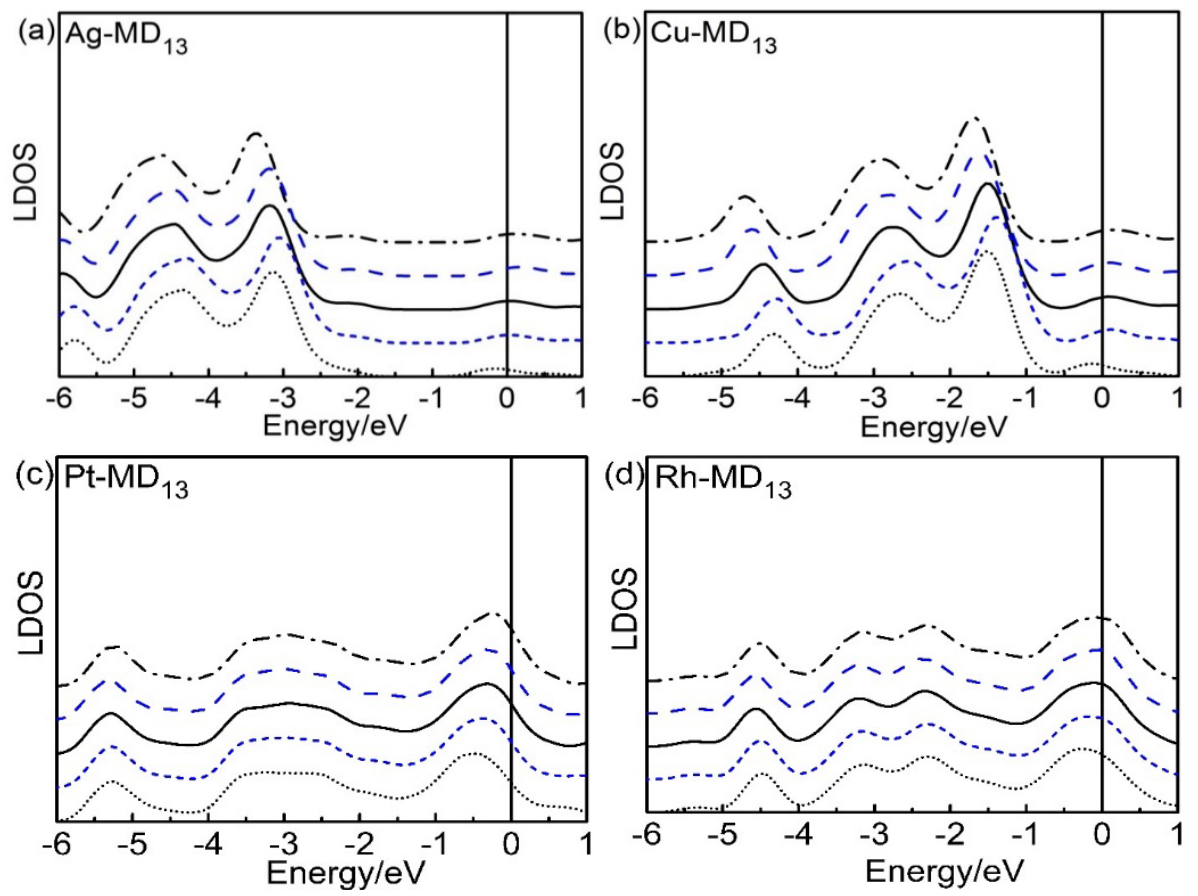

Figure S1. LDOS of $d$-band shown for $\mathrm{MD}_{13}$ structures. The black dash dot, blue dash, black solid, blue short dash, and short dot black indicate the excessive charge states including $(+2 e),(+1 e),(0),(-1 e)$, and $(-2 e)$, respectively. The Fermi level is considered at 0 .
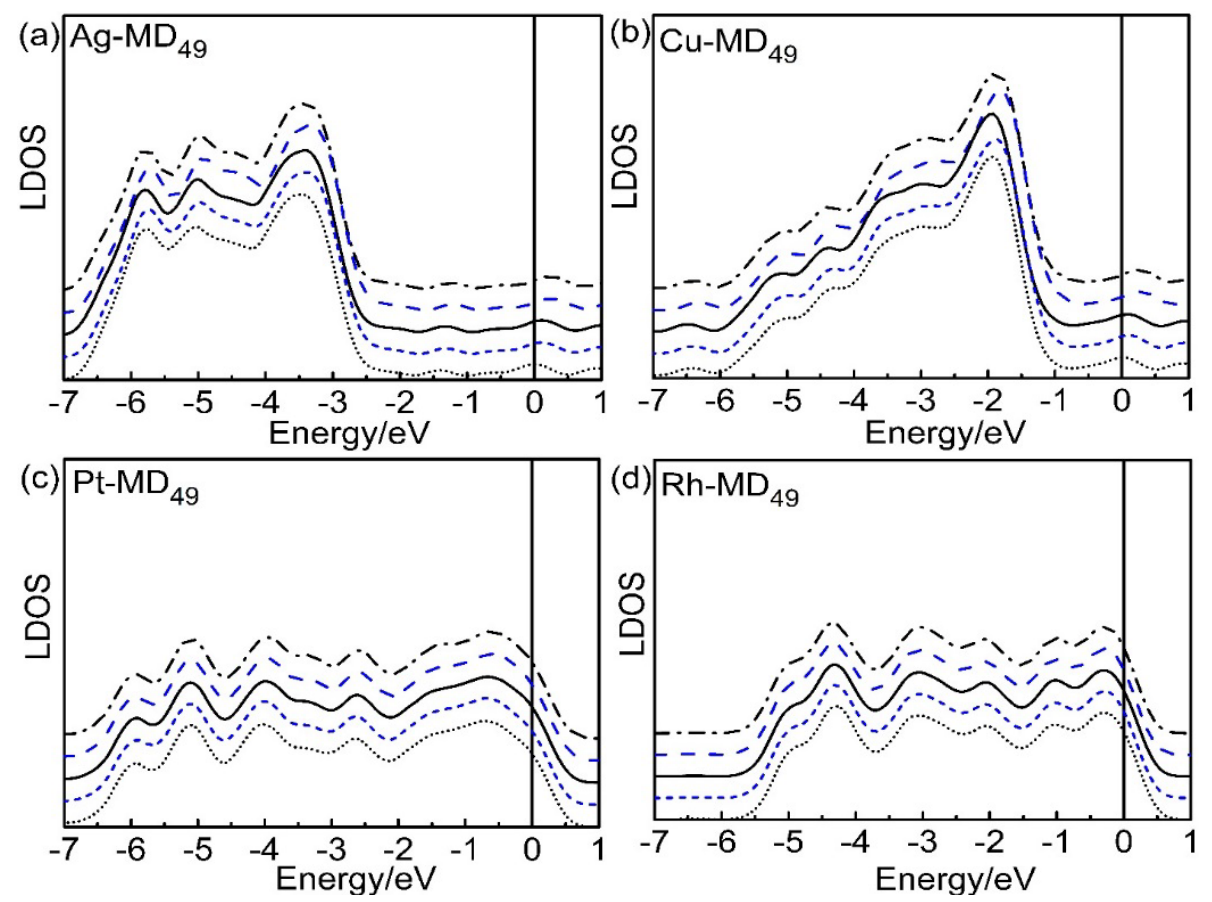

Figure S2. LDOS of $d$-band shown for $\mathrm{MD}_{49}$ structures. The black dash dot, blue dash, black solid, blue short dash, and short dot black indicate the excessive charge states including $(+2 e),(+1 e),(0),(-1 e)$, and $(-2 e)$, respectively. The Fermi level is considered at 0 . 

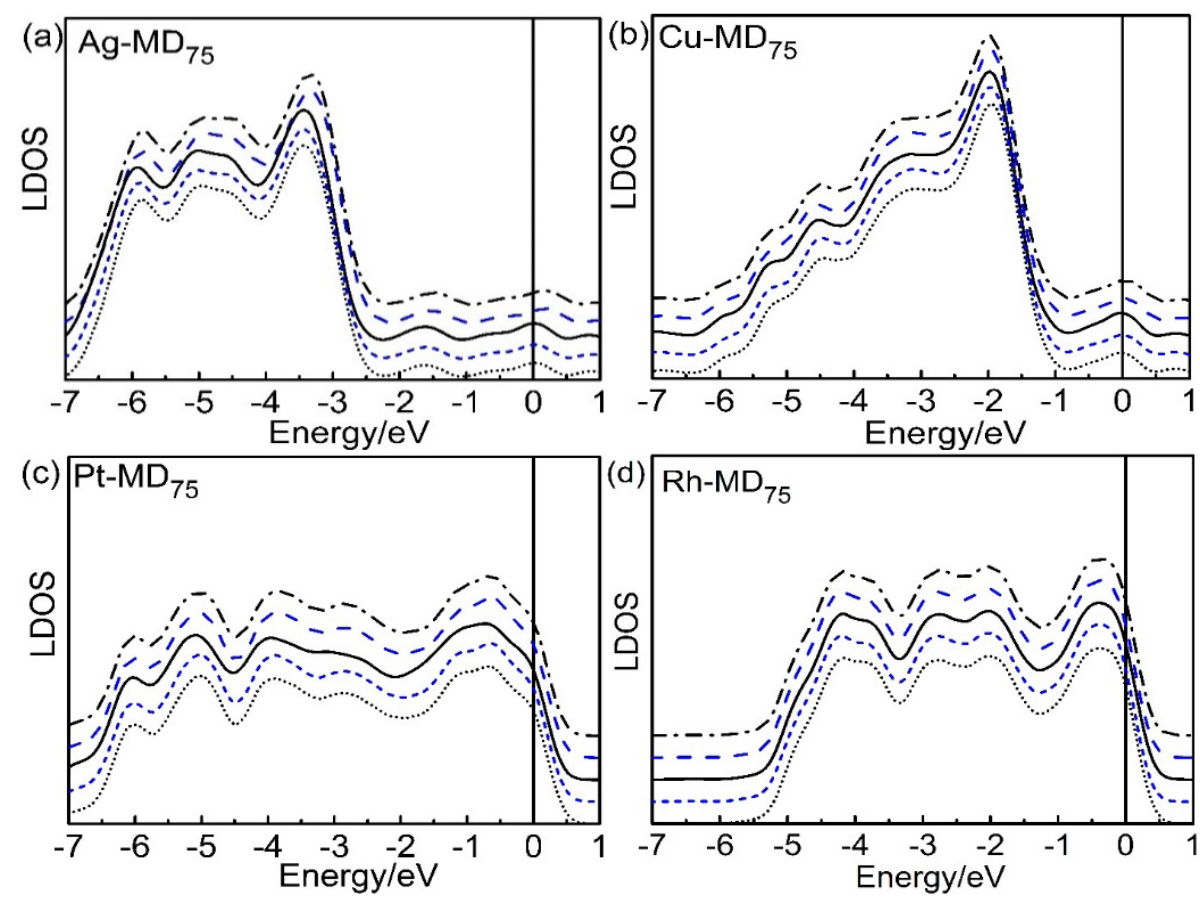

Figure S3. LDOS of $d$-band shown for $\mathrm{MD}_{75}$ structures. The black dash dot, blue dash, black solid, blue short dash, and short dot black indicate the excessive charge states including $(+2 e),(+1 e),(0),(-1 e)$, and $(-2 e)$, respectively. The Fermi level is considered at 0 .
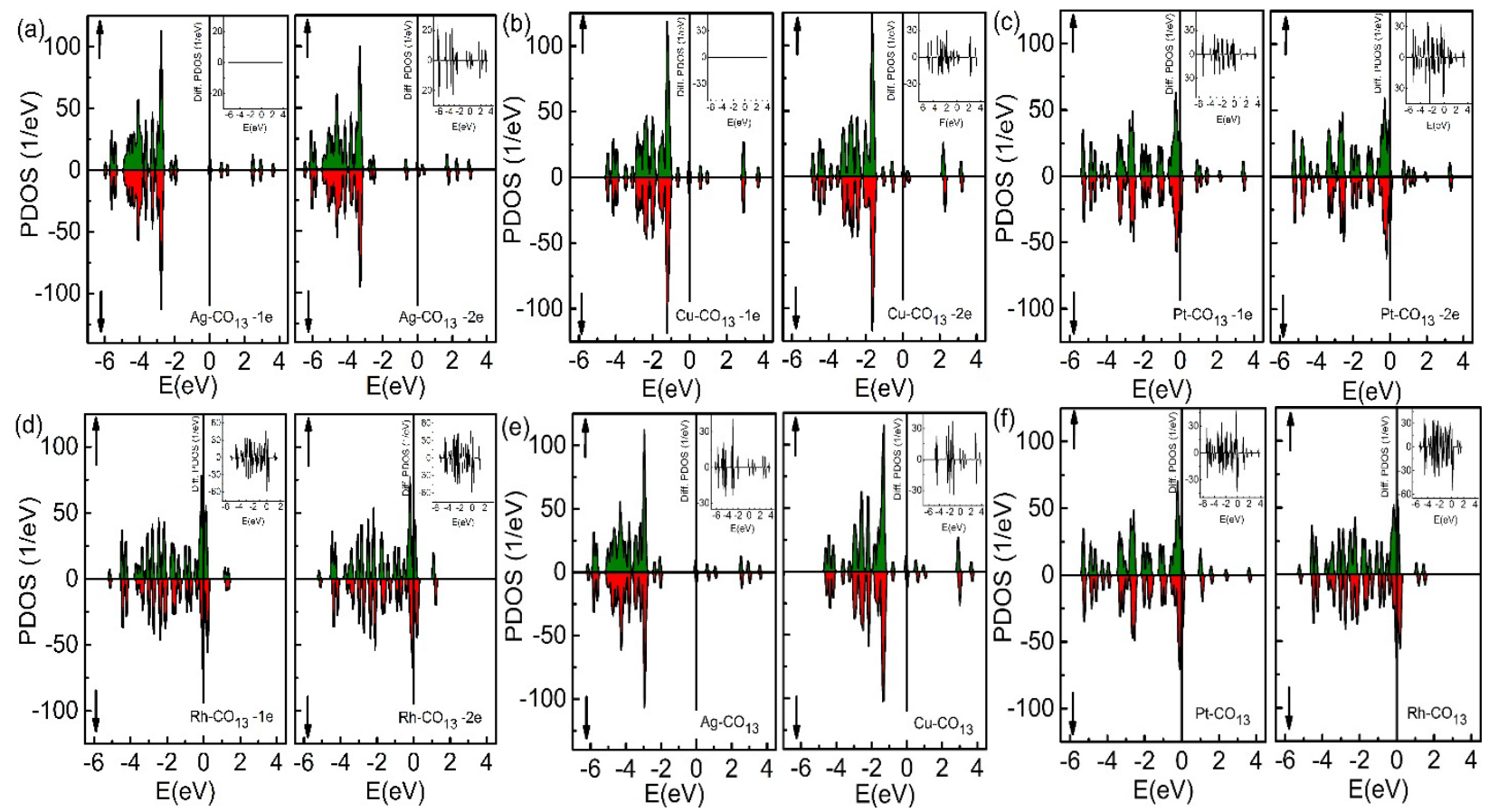

Figure S4. The spin-polarized PDOS of $4 d-\mathrm{Ag}, 3 d-\mathrm{Cu}, 5 d-\mathrm{Pt}$, and $4 d$-Rh for $\mathrm{CO}_{13}$ structures with (a-d) excessive charge states of $(-1 e)$ and $(-2 e)$ and (e-f) neutral charge state. Inset is the difference between spin-up and spin-down states in PDOS. The excessive charge states of (-1e) and (-2e) were considered in the singlet and double states for Ag and Cu nanoclusters, the doublet and triplet states for Pt nanoclusters, and the triplet and quartet states for Rh nanoclusters and the neutral charge state was considered in the double state for $\mathrm{Ag}$ and $\mathrm{Cu}$ nanoclusters, the triplet state for Pt nanoclusters, and the quartet state for Rh nanoclusters. The spin-up and spin-down configurations are indicated by the up and down arrows, respectively. The Fermi level is set at $0 \mathrm{eV}$. 

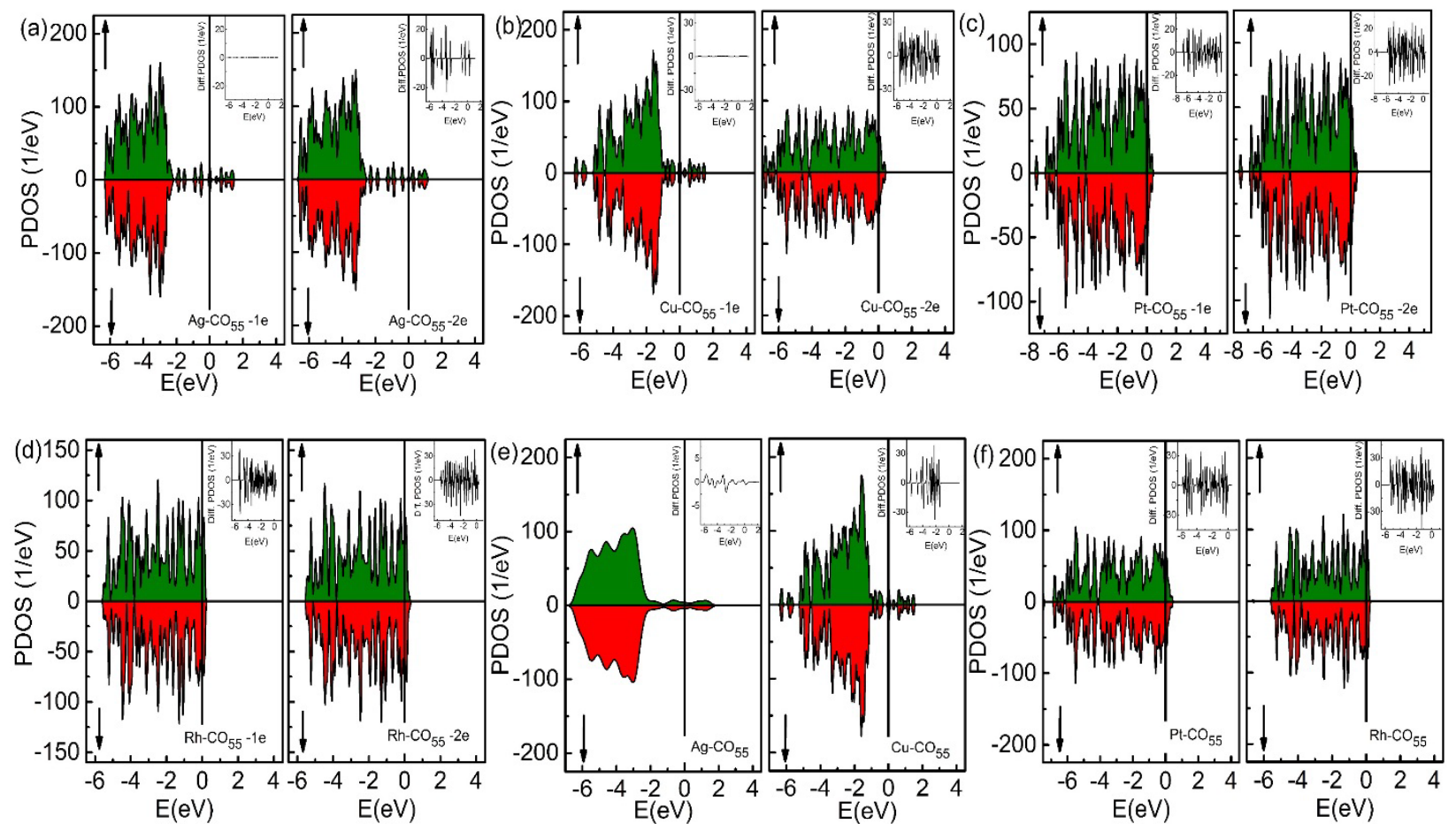

Figure S5. The spin-polarized PDOS of $4 d-\mathrm{Ag}, 3 d-\mathrm{Cu}, 5 d-\mathrm{Pt}$, and $4 d-\mathrm{Rh}$ for $\mathrm{CO}_{55}$ structures with (a-d) excessive charge states of $(-1 e)$ and $(-2 e)$ and (e-f) neutral charge state. Inset is the difference between spin-up and spin-down states in PDOS. The excessive charge states of (-1e) and (-2e) were considered in the singlet and double states for Ag and Cu nanoclusters, the doublet and triplet states for Pt nanoclusters, and the triplet and quartet states for Rh nanoclusters and the neutral charge state was considered in the double state for $\mathrm{Ag}$ and $\mathrm{Cu}$ nanoclusters, the triplet state for Pt nanoclusters, and the quartet state for Rh nanoclusters. The spin-up and spin-down configurations are indicated by the up and down arrows, respectively. The Fermi level is set at $0 \mathrm{eV}$. 

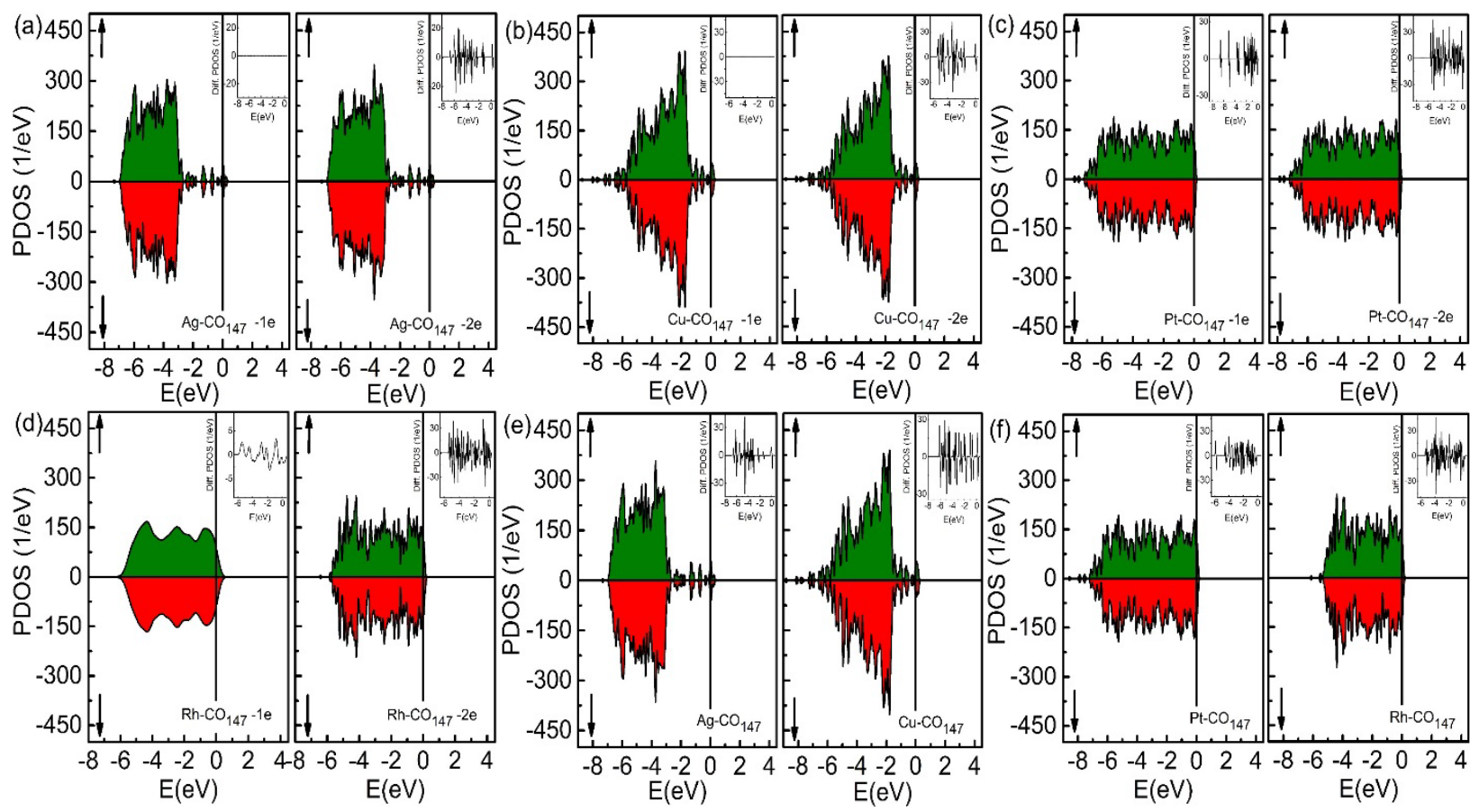

Figure S6. The spin-polarized PDOS of $4 d-\mathrm{Ag}, 3 d-\mathrm{Cu}, 5 d-\mathrm{Pt}$, and $4 d-\mathrm{Rh}$ for $\mathrm{CO}_{147}$ structures with (a-d) excessive charge states of $(-1 e)$ and $(-2 e)$ and (e-f) neutral charge state. Inset is the difference between spin-up and spin-down states in PDOS. The excessive charge states of (-1e) and (-2e) were considered in the singlet and double states for Ag and Cu nanoclusters, the doublet and triplet states for Pt nanoclusters, and the triplet and quartet states for Rh nanoclusters and the neutral charge state was considered in the double state for $\mathrm{Ag}$ and $\mathrm{Cu}$ nanoclusters, the triplet state for Pt nanoclusters, and the quartet state for $\mathrm{Rh}$ nanoclusters. The spin-up and spin-down configurations are indicated by the up and down arrows, respectively. The Fermi level is set at $0 \mathrm{eV}$. 

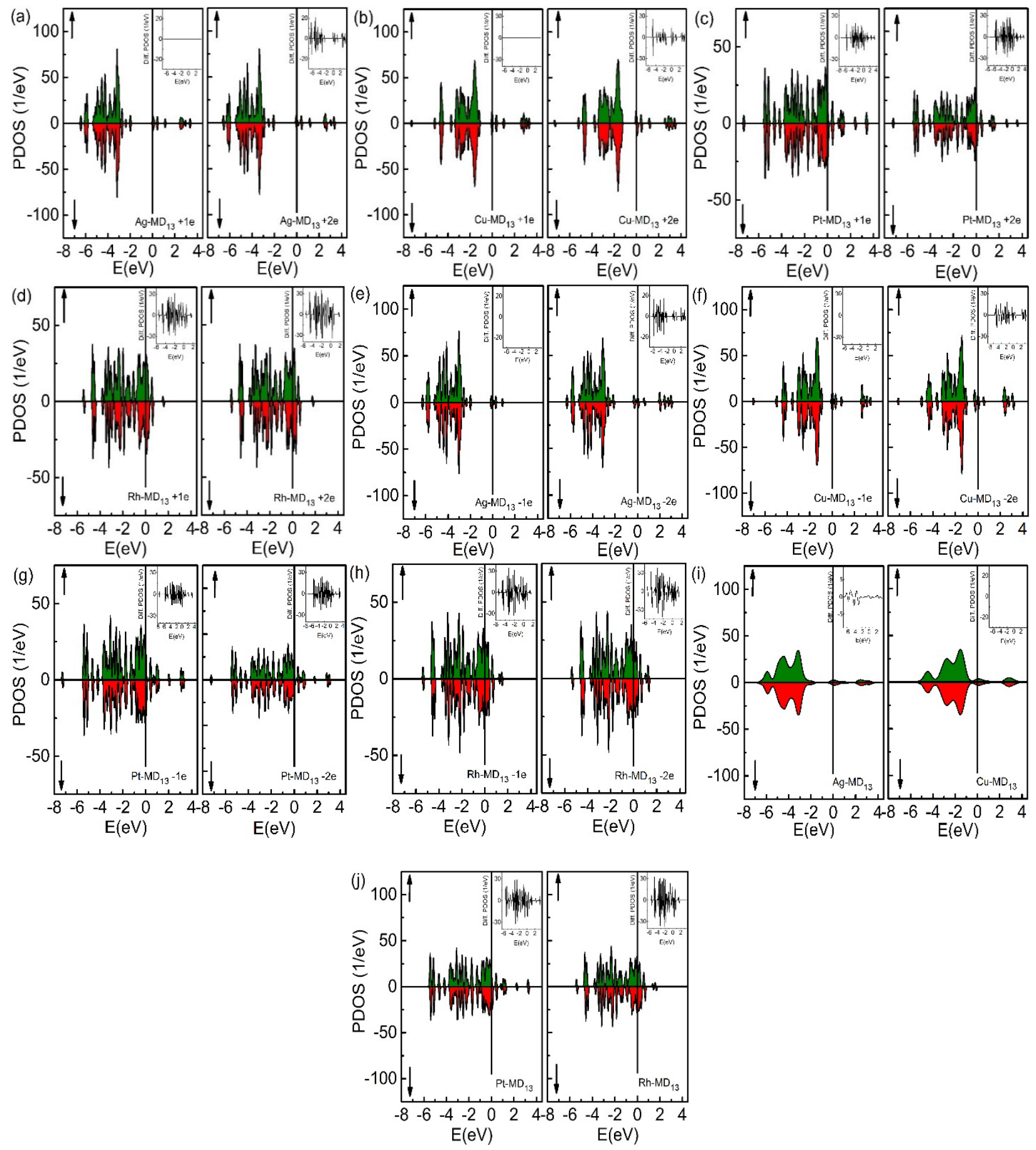

Figure S7. The spin-polarized PDOS of $4 d-\mathrm{Ag}, 3 d-\mathrm{Cu}, 5 d-\mathrm{Pt}$, and $4 d-\mathrm{Rh}$ for $\mathrm{MD}_{13}$ structures with (a-d) excessive charge states of $(+1 e)$ and $(+2 e),(e-h)$ excessive charge states of $(-1 e)$ and $(-2 e)$, and (i-j) neutral charge state. Inset is the difference between spin-up and spin-down states in PDOS. The excessive charge states of $( \pm 1 \mathrm{e})$ and $( \pm 2 \mathrm{e})$ were assumed in the singlet and double states for $\mathrm{Ag}$ and $\mathrm{Cu}$ nanoclusters, the doublet and triplet states for Pt nanoclusters, and the triplet and quartet states for Rh nanoclusters and the neutral charge state was considered in the double state for $\mathrm{Ag}$ and $\mathrm{Cu}$ nanoclusters, the triplet state for Pt nanoclusters, and the quartet state for Rh nanoclusters. The spin-up and spin-down configurations are indicated by the up and down arrows, respectively. The Fermi level is set at $0 \mathrm{eV}$. 

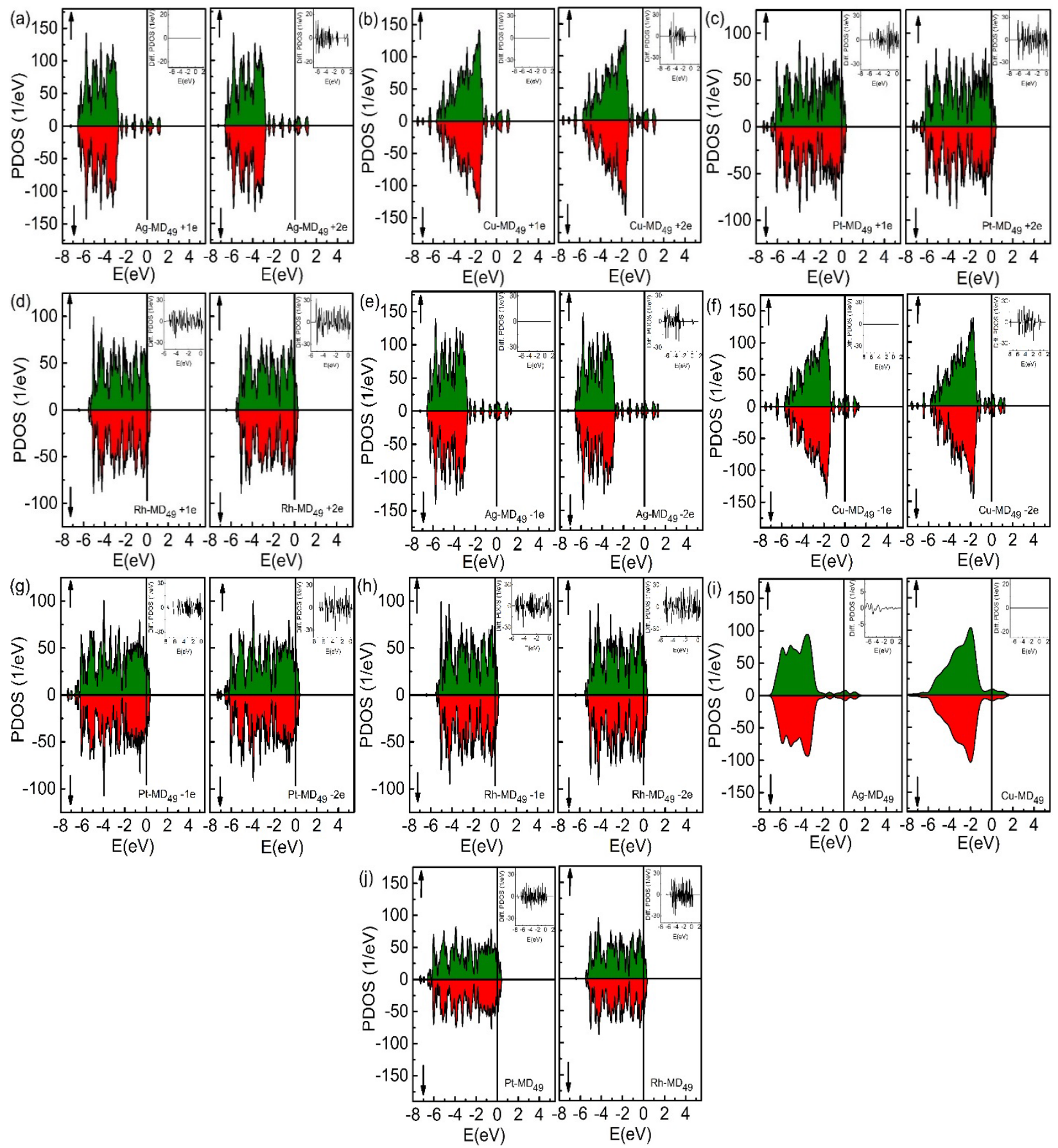

Figure S8. The spin-polarized PDOS of $4 d-\mathrm{Ag}, 3 d-\mathrm{Cu}, 5 d-\mathrm{Pt}$, and $4 d-\mathrm{Rh}$ for $\mathrm{MD}_{49}$ structures with (a-d) excessive charge states of $(+1 e)$ and $(+2 e),(e-h)$ excessive charge states of $(-1 e)$ and $(-2 e)$, and (i-j) neutral charge state. Inset is the difference between spin-up and spin-down states in PDOS. The excessive charge states of $( \pm 1 \mathrm{e})$ and $( \pm 2 \mathrm{e})$ were assumed in the singlet and double states for $\mathrm{Ag}$ and $\mathrm{Cu}$ nanoclusters, the doublet and triplet states for Pt nanoclusters, and the triplet and quartet states for Rh nanoclusters and the neutral charge state was considered in the double state for Ag and Cu nanoclusters, the triplet state for Pt nanoclusters, and the quartet state for Rh nanoclusters. The spin-up and spin-down configurations are indicated by the up and down arrows, respectively. The Fermi level is set at $0 \mathrm{eV}$. 

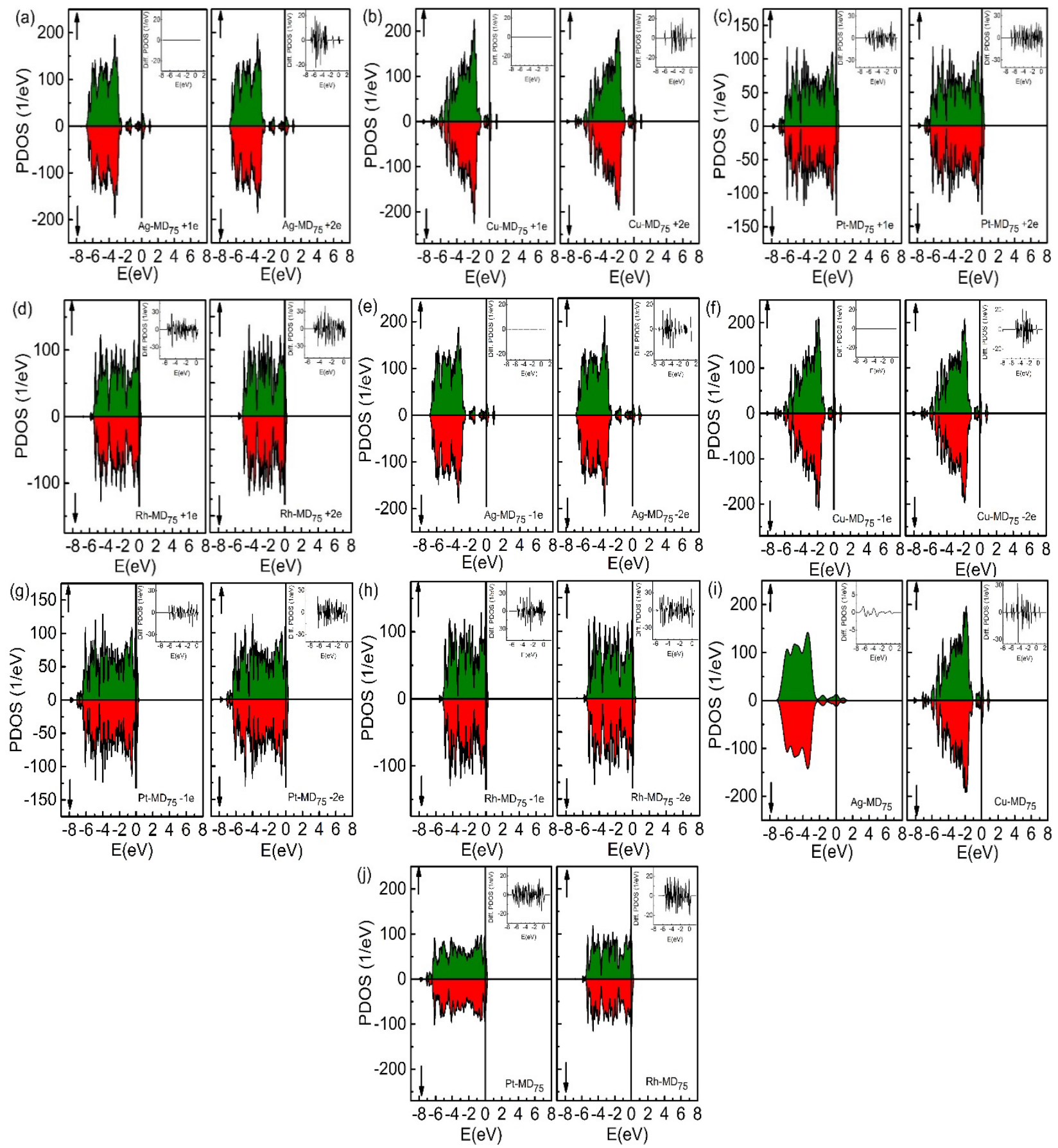

Figure S9. The spin-polarized PDOS of $4 d-\mathrm{Ag}, 3 d-\mathrm{Cu}, 5 d-\mathrm{Pt}$, and $4 d-\mathrm{Rh}$ for (a-d) $\mathrm{MD}_{75}$ structures with (a-d) excessive charge states of $(+1 e)$ and $(+2 e)$, (e-h) excessive charge states of $(-1 e)$ and $(-2 e)$, and $(i-j)$ neutral charge state. Inset is the difference between spin-up and spin-down states in PDOS. The excessive charge states of $( \pm 1 \mathrm{e})$ and $( \pm 2 \mathrm{e})$ were assumed in the singlet and double states for Ag and $\mathrm{Cu}$ nanoclusters, the doublet and triplet states for Pt nanoclusters, and the triplet and quartet states for $\mathrm{Rh}$ nanoclusters and the neutral charge state was considered in the double state for $\mathrm{Ag}$ and $\mathrm{Cu}$ nanoclusters, the triplet state for Pt nanoclusters, and the quartet state for Rh nanocluster. The spin-up and spin-down configurations are indicated by the up and down arrows, respectively. The Fermi level is set at $0 \mathrm{eV}$. 
Table S1. The calculated M-M bond length $d_{\mathrm{i}}(\AA)$ and charge of shell (e) at different position of $\mathrm{M}_{\mathrm{N}}$ metallic nanoclusters with MD structures and the excessive charge states from (+2e) to $(-2 e)$. The shell index counts the atomic layer from the outermost inwards.

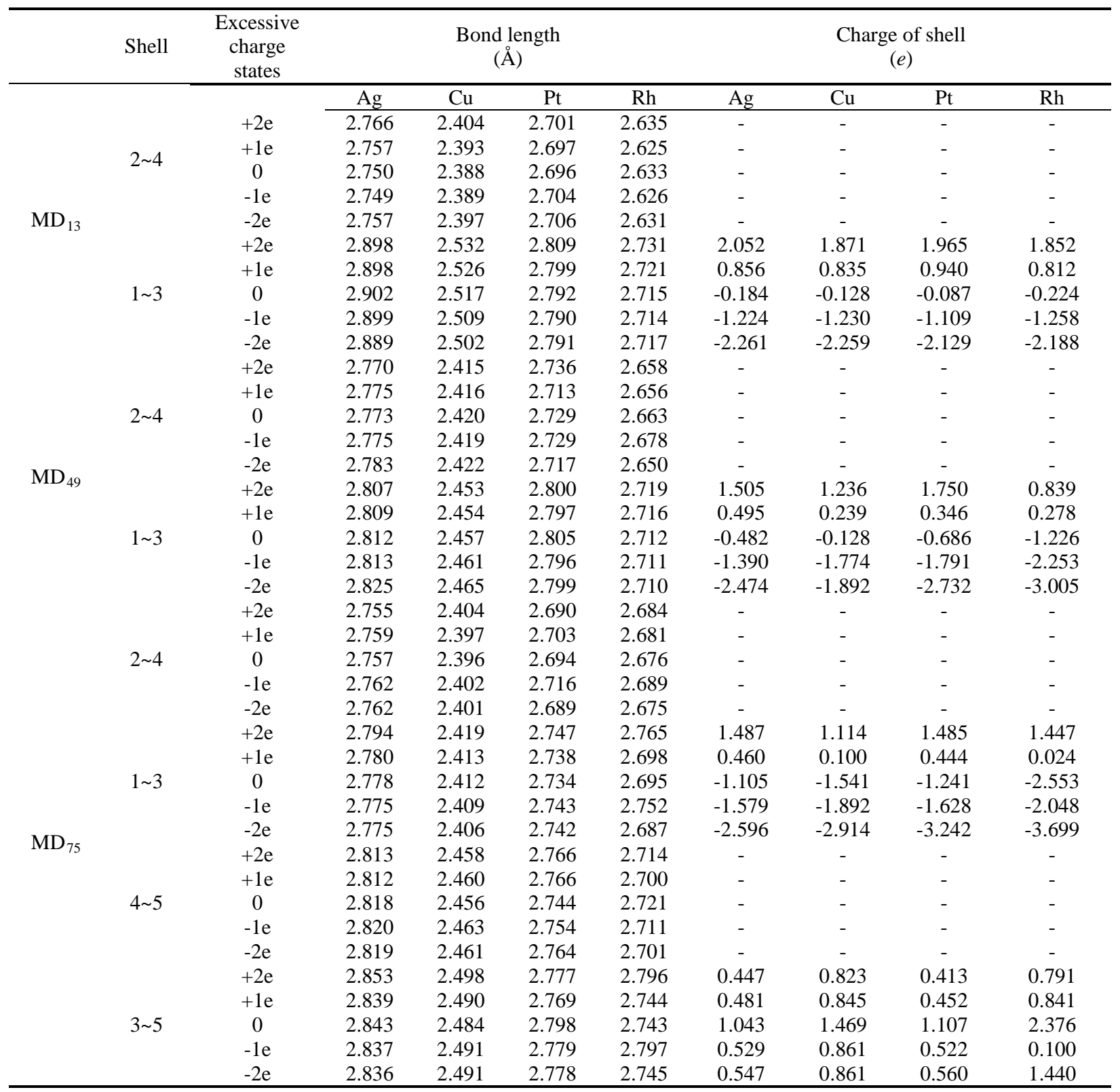


Table S2. Calculated gaps between spin-up and spin-down states of $\mathrm{M}_{\mathrm{N}}$ metallic nanoclusters with excessive charges. The magnetic behavior of $d$-band metallic nanoclusters happened in the positive (negative) even excessive charges for all metallic nanoclusters and the odd (positive/negative) excessive charges for Pt and Rh nanoclusters, while no magnetic behavior in the odd (positive/negative) excessive charges for Ag and $\mathrm{Cu}$ nanoclusters.

\begin{tabular}{|c|c|c|c|c|c|c|c|c|c|}
\hline & \multirow[t]{2}{*}{$\begin{array}{c}\text { Excessive charge } \\
\text { states } \\
\end{array}$} & \multicolumn{4}{|c|}{$\begin{array}{c}\text { Gap spin-up Alpha } \\
(\mathrm{eV})\end{array}$} & \multicolumn{4}{|c|}{$\begin{array}{c}\text { Gap spin-down Beta } \\
(\mathrm{eV})\end{array}$} \\
\hline \multirow{6}{*}{$\mathrm{CO}_{13}$} & & $\mathrm{Ag}$ & $\mathrm{Cu}$ & $\mathrm{Pt}$ & $\mathrm{Rh}$ & $\mathrm{Ag}$ & $\mathrm{Cu}$ & $\mathrm{Pt}$ & $\mathrm{Rh}$ \\
\hline & $+2 \mathrm{e}$ & 0.725 & 0.639 & 1.018 & 0.833 & 2.156 & 1.069 & 1.010 & 0.851 \\
\hline & $+1 \mathrm{e}$ & 0.702 & 0.610 & 1.048 & 0.954 & 0.702 & 0.610 & 1.044 & 0.958 \\
\hline & 0 & 0.695 & 0.611 & 0.058 & 0.976 & 0.688 & 0.597 & 1.005 & 0.982 \\
\hline & $-1 e$ & 0.687 & 0.598 & 0.190 & 0.992 & 0.687 & 0.598 & 0.892 & 0.995 \\
\hline & $-2 e$ & 0.689 & 0.598 & 0.233 & 0.993 & 0.706 & 0.615 & 0.302 & 0.968 \\
\hline \multirow{5}{*}{$\mathrm{CO}_{55}$} & $+2 \mathrm{e}$ & 0.386 & 0.331 & 0.181 & 0.099 & 0.510 & 0.393 & 0.182 & 0.067 \\
\hline & $+1 \mathrm{e}$ & 0.383 & 0.326 & 0.218 & 0.099 & 0.383 & 0.326 & 0.219 & 0.062 \\
\hline & 0 & 0.318 & 0.320 & 0.252 & 0.092 & 0.319 & 0.321 & 0.253 & 0.055 \\
\hline & $-1 e$ & 0.380 & 0.310 & 0.285 & 0.090 & 0.380 & 0.310 & 0.284 & 0.030 \\
\hline & $-2 \mathrm{e}$ & 0.357 & 0.263 & 0.307 & 0.071 & 0.248 & 0.305 & 0.308 & 0.041 \\
\hline \multirow{5}{*}{$\mathrm{CO}_{147}$} & $+2 \mathrm{e}$ & 0.251 & 0.194 & 0.009 & 0.019 & 0.252 & 0.196 & 0.062 & 0.027 \\
\hline & $+1 \mathrm{e}$ & 0.247 & 0.191 & 0.011 & 0.016 & 0.247 & 0.191 & 0.080 & 0.015 \\
\hline & 0 & 0.255 & 0.174 & 0.063 & 0.013 & 0.256 & 0.176 & 0.091 & 0.034 \\
\hline & $-1 e$ & 0.243 & 0.187 & 0.066 & 0.010 & 0.243 & 0.187 & 0.003 & 0.015 \\
\hline & $-2 e$ & 0.243 & 0.185 & 0.057 & 0.011 & 0.246 & 0.188 & 0.108 & 0.013 \\
\hline \multirow{5}{*}{$\mathrm{MD}_{13}$} & $+2 \mathrm{e}$ & 0.366 & 0.384 & 0.705 & 0.319 & 0.112 & 1.356 & 0.693 & 0.079 \\
\hline & $+1 \mathrm{e}$ & 0.123 & 0.342 & 0.554 & 0.330 & 0.123 & 0.342 & 0.549 & 0.006 \\
\hline & 0 & 0.301 & 0.317 & 0.224 & 0.334 & 0.137 & 0.309 & 0.416 & 0.105 \\
\hline & $-1 e$ & 0.256 & 0.295 & 0.225 & 0.335 & 0.256 & 0.295 & 0.227 & 0.336 \\
\hline & $-2 e$ & 0.572 & 0.487 & 0.914 & 0.332 & 0.242 & 0.295 & 0.227 & 0.012 \\
\hline \multirow{5}{*}{$\mathrm{MD}_{49}$} & $+2 \mathrm{e}$ & 0.118 & 0.185 & 0.453 & 0.067 & 0.452 & 0.318 & 0.451 & 0.013 \\
\hline & $+1 \mathrm{e}$ & 0.114 & 0.185 & 0.413 & 0.068 & 0.114 & 0.185 & 0.412 & 0.057 \\
\hline & 0 & 0.134 & 0.171 & 0.363 & 0.068 & 0.128 & 0.175 & 0.361 & 0.022 \\
\hline & $-1 e$ & 0.138 & 0.159 & 0.335 & 0.061 & 0.138 & 0.159 & 0.334 & 0.053 \\
\hline & $-2 e$ & 0.102 & 0.118 & 0.278 & 0.036 & 0.146 & 0.150 & 0.293 & 0.032 \\
\hline \multirow{5}{*}{$\mathrm{MD}_{75}$} & $+2 \mathrm{e}$ & 0.003 & 0.189 & 0.291 & 0.054 & 0.213 & 0.187 & 0.034 & 0.063 \\
\hline & $+1 \mathrm{e}$ & 0.015 & 0.193 & 0.255 & 0.014 & 0.015 & 0.193 & 0.054 & 0.037 \\
\hline & 0 & 0.128 & 0.232 & 0.187 & 0.028 & 0.041 & 0.231 & 0.054 & 0.052 \\
\hline & $-1 e$ & 0.131 & 0.221 & 0.190 & 0.027 & 0.131 & 0.221 & 0.090 & 0.021 \\
\hline & $-2 \mathrm{e}$ & 0.122 & 0.239 & 0.157 & 0.022 & 0.128 & 0.238 & 0.103 & 0.030 \\
\hline
\end{tabular}

